\title{
ALGUNS MODELOS CLÁSSICOS PARA PREVISÃO DOS PREÇOS DO PETRÓLEO BRUTO NO MERCADO INTERNACIONAL
}

\author{
Andre Assis de Salles \\ Universidade Federal do Rio de Janeiro / Escola Politécnica \\ Centro de Tecnologia - Bloco F - sala F101- Ilha do Fundão - Rio - Brasil \\ as@ufrj.br \\ Mariana Alves Londe \\ Universidade Federal do Rio de Janeiro / Escola Politécnica \\ mlonde@poli.ufrj.br
}

\section{RESUMO}

Este trabalho tem como propósito identificar a adequação de modelos de previsão clássicos para os preços do petróleo bruto dos tipos Brent e WTI, principais referências de preços do mercado internacional. Para se atingir esse objetivo foram elaborados modelos de previsão do tipo ARIMA e modelos vetoriais autoregressivos (VAR) para séries temporais de retornos diários e semanais das cotações dos preços das duas referências estudadas. Para construção desses modelos foram verificadas as hipóteses da violação dos pressupostos de normalidade e de estacionariedade. Os resultados obtidos indicam que, em geral, esses modelos se mostram adequados previsão dos preços do petróleo bruto. Os dados primários utilizados foram informações em um período posterior a deflagração da crise financeira internacional do subprime.

Palavra-chave: Preço do Petróleo; Modelos ARIMA; Modelos VAR.

\begin{abstract}
The purpose of this paper is to identify the adequacy of classical forecast models for Brent and WTI crude oil prices, the main international market price benchmarks. In order to achieve this objective, ARIMA forecast models and vector autoregressive models were prepared for daily and weekly price returns time series of the two benchmarks studied. For the construction of these models, the hypothesis of violation of normality and stationarity assumptions was verified. The results indicate, in general, these models are suitable for predicting crude oil prices. The primary data used were crude oil daily quotes in a period subsequent to the outbreak of the subprime international financial crisis.
\end{abstract}

Keywords: Crude Oil Prices; ARIMA Models; VAR Models.

\section{Como Citar:}

SALLES, A. A.; LONDE, M. A.. Alguns Modelos Clássicos para Previsão dos Preços do Petróleo Bruto no Mercado Internacional. In: SIMPÓSIO DE PESQUISA OPERACIONAL E LOGÍSTICA DA MARINHA, 19., 2019, Rio de Janeiro, RJ. Anais [...]. Rio de Janeiro: Centro de Análises de Sistemas Navais, 2019. 


\section{INTRODUÇÃO}

O petróleo possui papel relevante em todos os setores da economia, do agrícola ao farmacêutico passando pelo industrial e energético. É conhecida a relação entre o consumo de petróleo e seus derivados e o crescimento da economia de um país, o que torna a conhecimento do comportamento dos preços do petróleo bruto crucial tanto para produtores quanto para compradores.

Presente na cadeia de produção de inúmeros produtos, a expectativa dos preços de petróleo é fundamental para tomadores de decisões de políticas públicas, assim como para tomadores decisão nas empresas que dependem direta ou indiretamente das expectativas dos preços do petróleo. Além disso, para os agentes econômicos atuantes no mercado de energia e, especialmente, no mercado de petróleo essas expectativas indispensáveis. No mercado de petróleo em períodos passados foi comum a regulação dos preços do petróleo. Braginskii (2009) divide a história do petróleo em quatro períodos e somente no último período, o período entre 1986 e os dias atuais, não se observa regulação de preços. Com o desenvolvimento dos mercados e, em especial do mercado de derivativos, o petróleo bruto passa a ter um comportamento semelhante ao de um ativo financeiro. Negociado em bolsas de valores, o petróleo bruto é um ativo cada vez mais frequente em carteiras de investimento diversificadas no mercado internacional. Com isto, o preço do petróleo é sensível às variáveis macroeconômicas como um ativo financeiro com variações diárias e alta volatilidade. Aumentando, ainda mais, a complexidade da obtenção das expectativas de preços dessa commodity.

Desse modo a busca por modelos de previsão de preços adequados é um tópico frequente na literatura de energia e de métodos quantitativos. Pode se destacar os artigos de Behmeri e Manso (2013) e de Frey et al. (2009) que apresentam uma revisão de diversos métodos estatísticos utilizados para a previsão dos preços do petróleo bruto. Enquanto Worthington (2012), por sua vez, apresentou um estudo sobre pesquisas em finanças relacionadas com o petróleo bruto.

Este trabalho tem como propósito estudar a adequação de modelos de previsão do tipo ARIMA, modelos autoregressivos integrados de médias móveis e de modelos vetoriais autoregressivos (VAR), para a obtenção de expectativas de preços do petróleo dos tipos Brent e WTI no curto prazo.

Este trabalho está estruturado em seis seções. Além desta introdução na próxima seção é apresentado um breve resumo da revisão bibliográfica feita para elaboração deste trabalho. Enquanto a Seção 3 descreve os métodos econométricos utilizados, a saber: modelos ARIMA e modelos vetoriais autoregressivos. A amostra utilizada está descrita na Seção 4. Enquanto os resultados obtidos e os comentários finais são tratados, respectivamente, na seções 5 e 6 . Por fim estão listados as referências bibliográficas utilizadas.

\section{REVISÃO BIBLIOGRÁFICA - UM RESUMO}

A procura de modelos adequados para previsão dos preços de commodities e, em particular, do petróleo é um tema recorrente na literatura de séries temporais. Assim como é usual o relato da dificuldade de se prever acuradamente os preços do petróleo e de outras commodities dada a alta volatilidade dos respectivos mercados. São muitos estudos e pesquisas apresentados na literatura que procuram estabelecer modelos de previsão mais adequados para preços de commodities. Nesses trabalhos os modelos do tipo ARIMA e da classe ARCH são os mais utilizados. A Tabela 1 adiante apresenta um sumário com trabalhos mais recentes sobre o tema, que foram objeto de um levantamento bibliográfico para elaboração deste trabalho. 
A Tabela 1 mostra que a procura por modelos mais adequados e precisos para preços de commodities é uma linha de pesquisa bem difundida. Os diversos tipos de modelos utilizados deixam claro que é uma busca complexa, e divergente ao se considerar fatores relevantes para a formação dos preços. Como mencionado, os modelos do tipo ARIMA e do tipo ARCH se mostram presentes e com maior frequência na literatura como candidatos a modelos mais adequados para a obtenção de expectativas de preços de commodities.

Tabela 1 - Resumo da Revisão Bibliográfica

\begin{tabular}{|c|c|c|c|c|c|}
\hline № & Autor(es) & $\begin{array}{c}\text { Data } \\
\text { Publicação } \\
\end{array}$ & Commodity & Amostra - Período & $\begin{array}{c}\text { Método } \\
\text { Utilizado }\end{array}$ \\
\hline 1 & Assis et al. & 2010 & Cacau & $\begin{array}{c}\text { Dados Mensais } \\
(01 / 1992-12 / 2006)\end{array}$ & $\begin{array}{l}\text { - Alisamento } \\
\text { Exponencial } \\
\text { - ARIMA } \\
\text { - GARCH }\end{array}$ \\
\hline 2 & Nadarajah et al. & 2014 & $\begin{array}{l}\text { Cacau } \\
\text { Petróleo Brent } \\
\text { Petróleo WTI } \\
\text { Ouro } \\
\text { Prata } \\
\end{array}$ & $\begin{array}{c}\text { Dados Diários } \\
(12 / 03 / 1993-13 / 03 / 2013)\end{array}$ & - GARCH \\
\hline 3 & Klein e Walther & 2016 & $\begin{array}{l}\text { Petróleo Brent } \\
\text { Petróleo WTI }\end{array}$ & $\begin{array}{c}\text { Dados Diários } \\
(01 / 01 / 1995-31 / 12 / 2014)\end{array}$ & - MMGARCH \\
\hline 4 & Wang et al. & 2017 & Petróleo WTI & $\begin{array}{c}\text { Dados Mensais } \\
(01 / 1992-12 / 2015)\end{array}$ & $\begin{array}{l}\text { - Modelos } \\
\text { Combinados }\end{array}$ \\
\hline 5 & Funk & 2018 & Petróleo Brent & $\begin{array}{c}\text { Dados Mensais } \\
(06 / 1982-11 / 2017)\end{array}$ & $\begin{array}{l}\text { - Modelos } \\
\text { Combinados } \\
\text { - ARMA } \\
\text { - VAR } \\
\end{array}$ \\
\hline 6 & Herrera et al. & 2018 & Petróleo WTI & $\begin{array}{c}\text { Dados Diários }(02 / 01 / 2007 \\
-02 / 04 / 2015)\end{array}$ & $\begin{array}{l}\text { - Risk Metrics } \\
\text { - GARCH } \\
\text { - FIGARCH } \\
\text { - MSGARCH } \\
\text { - EGARCH } \\
\text { - GJR-GARCH } \\
\end{array}$ \\
\hline 7 & Dbouk e Jamali & 2018 & Petróleo WTI & $\begin{array}{c}\text { Dados Diários entre } \\
(02 / 01 / 1990-01 / 01 / 2010)\end{array}$ & $\begin{array}{l}\text { - Modelos } \\
\text { Lineares } \\
\text { - ARMA } \\
\text { - ECM } \\
\text { - ARDL } \\
\text { - Redes Neurais }\end{array}$ \\
\hline
\end{tabular}

Este trabalho se diferencia dos aqui relacionados no que se refere ao período estudado. Deve se ressaltar a novidade deste trabalho que se utiliza de dados pós crise do subprime deflagrada em 2008 que atingiu a economia mundial e, principalmente, os mercados de capitais e de créditos. A metodologia adotada para elaboração deste trabalho está descrita na próxima seção.

\section{ABORDAGEM METODOLÓGICA}

No estudo de uma série temporal dois pressupostos se fazem necessários na estimação dos modelos: a normalidade das informações em questão ou dos termos estocásticos relacionados nos modelos a serem estimados; e a estacionariedade das séries temporais de interesse envolvidas nos modelos a serem estimados. Assim, além de um resumo estatístico com medidas de posição e de dispersão, foram testadas as hipóteses de normalidade e de estacionariedade de todas as series temporais utilizadas neste trabalho. Para os testes de normalidade foi utilizado o teste de Jarque e Bera, enquanto para os testes 
de estacionariedade o teste utilizado foi o de raízes unitárias de Dickey e Fuller Aumentado, teste ADF, conforme a descrição disponível em Gujarati e Porter (2011).

\subsection{Estacionariedade e Cointegração}

Para o estudo de uma série temporal, é necessário que a variância e média não variem ao longo do tempo, e a covariância entre dois períodos de tempo deve depender apenas da defasagem entre esses dois e não do tempo real em que ela é computada. Um processo estocástico com esta característica é dito fracamente estacionário, o que é o bastante para a maioria das situações. Contudo, em geral, dados temporais raramente possuem essa característica. Nestes casos, é necessário que se transforme a série em uma nova série derivada e estacionária. Para isso, é preciso, primeiro, identificar se a série é estacionária ou não, o que pode ser feito de diversas formas. O teste de raiz unitária é o mais difundido e utilizado para isso. Esse teste, como descrito por Gujarati e Porter (2011), possui como ponto de partida a equação (1) a seguir, no qual $u_{t}$ é um termo estocástico, erro ou ruído branco, ou seja, é puramente aleatório, com média zero variância constante $\sigma^{2} \mathrm{e}$ serialmente não correlacionado.

$$
Y_{t}=\rho Y_{t-1}+u_{t}-1 \leq \rho \leq 1
$$

No caso em que o parâmetro $\rho$ tem o valor igual a 1, temos que a equação representa um modelo de passeio aleatório. Este é não-estacionário, uma vez que, enquanto sua média será igual ao valor de $Y_{t}$, sua variância terá o valor de $t \sigma^{2}$, que varia com o tempo. Logo, se for possível comprovar estatisticamente que o valor de $\rho$ é 1 , temos assim que a série temporal não é estacionária. Contudo, o habitual teste $t$ de Student, para se testar hipóteses relacionadas aos parâmetros do modelo, não é o ideal para esta situação, sendo assim necessário manipular a equação até obter a expressão (4) abaixo.

$$
\begin{aligned}
& Y_{t}-Y_{t-1}=\rho Y_{t-1}-Y_{t-1}+u_{t} \\
& \Delta Y_{t}=(\rho-1) Y_{t-1}+u_{t} \\
& \Delta Y_{t}=\delta Y_{t-1}+u_{t}
\end{aligned}
$$

Desse modo, se verifica a hipótese nula do $\delta=(\rho-1)$ ser zero, com a hipótese alternativa de ser negativo. Ainda, é demonstrado que, sobre a hipótese nula de que $\delta=0$, a estatística $t$ do teste segue uma distribuição $\tau$, sugerida para o teste de Dickey-Fuller (DF). Para se realizar um teste DF, é necessário também ver outras características. É possível que a distribuição possua deslocamento, ou tendências estocásticas ou determinísticas. Desse modo, o teste deve ser estimado de três diferentes formas, além do teste realizado em (5).

$$
\Delta Y_{t}=\delta Y_{t-1}+u_{t}
$$

Outros dois modelos devem ser utilizados para testar a hipótese da estacionariedade, como descritos em (6) e (7), respectivamente, com deslocamento e com deslocamento em torno de uma tendência determinística.

$$
\begin{aligned}
& \Delta Y_{t}=\beta_{1}+\delta Y_{t-1}+u_{t} \\
& \Delta Y_{t}=\beta_{1}+\beta_{2} t+\delta Y_{t-1}+u_{t}
\end{aligned}
$$

Assim, é necessário que sejam estimados os valores de $\delta$ para as três possibilidades de modelos, para então observar qual o caso em que a série se encontra, e se ela é ou não estacionária. Deve-se acrescentar que o termo $u_{t}$ é não correlacionado por suposição. 
De acordo com Gujarati e Porter (2011), neste teste estendem-se as três equações ao acrescentar valores defasados da variável dependente. Nos casos em que isso não ocorre, deve-se utilizar o teste de Dickey-Fuller Aumentado (DFA), conforme mostrada na equação (8) a seguir.

$$
\Delta Y_{t}=\beta_{1}+\beta_{2} t+\delta Y_{t-1}+\sum_{i=1}^{m} \alpha_{1} \Delta Y_{t-i}+\varepsilon_{t},
$$

onde o parâmetro $m$ é definido como a quantidade de termos para que o erro - dado por $\varepsilon_{t}$, sendo um ruído branco puro - seja não correlacionado. Deve-se lembrar que se procura testar o valor de $\delta$, e se utiliza a mesma distribuição $\tau$ do teste DF para isso. Nestes testes, uma vez que a hipótese nula é de que a série apresenta uma raiz única, um valor p alto irá indicar que a série é não estacionária, enquanto um próximo de zero demonstra estacionariedade.

\subsection{Verificação da Cointegração}

Ao procurar uma relação entre duas variáveis pode ocorrer uma regressão espúria, ou seja, uma relação entre as duas variáveis mas não é verdadeira quando se observa a realidade, ou estatisticamente não significante. Esse fenômeno pode ocorrer quando se estima um modelo de regressão com variáveis não estacionárias. Contudo, se as séries compartilharem uma mesma tendência, a regressão entre as duas pode não ser espúria (ver Gujarati e Porter (2011)). Considerando duas variáveis $X$ e $Y$, ambas séries temporais cujas primeiras diferenças são estacionárias, a combinação linear das duas variáveis, como mostra a equação (9), deveria ser estacionária, pois supostamente isto elimina a tendência estocástica que causa a não estacionariedade.

$$
\mu_{t}=Y_{t}-\beta_{1}-\beta_{2} X_{t}
$$

Neste caso, a regressão entre as duas variáveis não seria espúria e pode-se dizer que essas variáveis são cointegradas. $\mathrm{Na}$ equação (9) acima, conhecida como regressão de cointegração, o $\beta_{2}$ é chamado de parâmetro de cointegração, e ela pode ser estendida para $k$ modelos, no caso em que teríamos $k$ parâmetros. Ainda de acordo com Gujarati e Porter (2011), a existência de cointegração pode evitar regressões espúrias de modo que é necessário que se possa identificar a existência da mesma. Como a verificação da cointegração depende da estacionariedade dos resíduos $\mu_{t}$ do modelo exibido na equação (9) acima, assim pode-se aplicar o teste de Dickey-Fuller ou Dickey-Fuller Aumentado na série dos resíduos $\mu_{t}$. Contudo, uma vez que os parâmetros $\beta$ 's são estimados, os valores críticos, para se testar a significância dos parâmetros dos modelos DF ou DFA não são adequados. Engle e Granger calcularam os valores adequados para essa aplicação do teste, a aplicação do teste de raiz única de Dickey-Fuller para os resíduos expressados por uma regressão de cointegração, designada como teste de Engle-Granger (EG) ou, em sua variação, EngleGranger Aumentado (EGA).

A seguir são descritos os modelos estimados para o desenvolvimento deste trabalho, após os testes necessários para proceder a estimação de modelos ARIMA univariados.

\subsection{Modelos ARIMA Univariados}

Com a identificação da estacionariedade da série é possível procurar o melhor modelo. Desse modo as expectativas das variações dos preços do petróleo, dos tipos Brent e WTI, foram estimadas através de modelos univariados do tipo ARIMA -- autoregressivos integrados com média móvel. Os modelos ARIMA identificam os valores da variável dependente relacionados com seus próprios valores passados tendo como base a ideia de "deixar os dados falarem por si mesmos", como observa Gujarati e Porter (2011). Esses 
modelos são ditos ateóricos, pois não consideram a teoria econômica tradicional.

$\mathrm{Na}$ descrição de um modelo ARIMA inicialmente deve-se definir a primeira parte, ou seja, um modelo autoregressivo de p-ésima ordem, designado como AR(p), que expressa a explicação do valor atual de $Y_{t}$ através de uma proporção dos $\mathrm{p}$ valores anteriores de $Y_{t}$ acrescido de um choque aleatório, e pode ser descrito pela expressão (10) a seguir.

$$
Y_{t}=\alpha_{0}+\alpha_{1} Y_{t-1}+\alpha_{2} Y_{t-2}+\cdots+\alpha_{p} Y_{t-p}+u_{t}
$$

Enquanto na outra parte do modelo ARIMA, ou do modelo ARMA, corresponde ao modelo de média móvel da variável $Y_{t}$ pode ser explicada por outro mecanismo, onde a variável $Y_{t}$ é expressa por uma combinação dos $q$ termos de erros, ou choques aleatórios, anteriores acrescido de um termo constante. Assim um modelo de médias móveis de $q$ ésima ordem, ou $\operatorname{MA}(q)$, pode ser descrito pela expressão (11) mostrada a seguir.

$$
Y_{t}=\mu+\beta_{0} u_{t}+\beta_{1} u_{t-1}+\cdots+\beta_{q} u_{t-q}
$$

Um modelo $\operatorname{ARMA}(p, q)$ irá possuir $p$ termos autoregressivos e $q$ termos relacionados às médias móveis, e pode ser descrito, em sua forma mais simples um modelo $\operatorname{ARMA}(1,1)$, através da equação (12) abaixo.

$$
Y_{t}=\theta+\alpha_{1} Y_{t-1}+\beta_{0} u_{t}+\beta_{1} u_{t-1}
$$

Deve-se observar que os modelos são adequados para séries temporais estacionarias. Se esse pressuposto for violado é necessário que se efetue a transformação da série temporal em uma série temporal estacionária o que é possível ao se diferenciar a série temporal. Nesse caso o modelo $\operatorname{ARMA}(p, q)$ após a variável de interesse $Y_{t}$ ser diferenciada $\mathrm{d}$ vezes torna-se um modelo ARIMA, um modelo ARIMA $(p, d, q)$ com $p$ termos e $q$ termos de médias móveis.

Para a obtenção das características do modelo, ou dos valores de $p$ e $q$, classicamente se utiliza as funções de autocorrelação (FAC) e de autocorrelação parcial (FACP) da série temporal de interesse, como descrito por Fava (2000). No entanto, em geral, não é trivial identificar se o comportamento de uma serie temporal segue as características da FAC e da FACP para identificação de modelos ARIMA. Essas características da FAC e da FACP podem ser sumarizadas da seguinte forma: declinante e truncada em $k=p$, truncada em $k=q$ e declinante, declinante e declinante, respectivamente, um processo $\operatorname{AR}(p)$, um processo $\operatorname{MA}(q)$ e processo $\operatorname{ARMA}(p, q)$. A dificuldade para se identificar o comportamento de uma série, ou se verificar se essa segue características conhecidas, conduz a outro procedimento para que se faça a identificação dos graus de $p$ e $q$. Nesse processo, ao invés de se estabelecer com precisão esses parâmetros, são elaborados modelos correspondentes a vários pares $(p, q)$, e escolhe-se o modelo utilizando-se algum critério de seleção de modelos.

\subsection{Modelos Vetoriais Autoregressivos - Modelos VAR}

Em um segundo momento este trabalho procura verificar as interrelações entre as séries temporais de variação dos preços do petróleo bruto das referências Brent e WTI. Assim, é necessário apresentar aqui, também, os modelos vetoriais autoregressivos (VAR). Esses modelos levam em consideração a interferência de uma variável no valor da outra variável e vice-versa, de modo que é possível utilizar uma outra variável explicativa dentro do modelo autoregressivo. O modelo procura explicar e prever suas variáveis a partir das 
equações estimadas, e sua maior dificuldade está na estimação do número de defasagens de cada variável a ser utilizada no modelo.

Neste modelo bivariado, consideram-se todas as variáveis envolvidas como endógenas (ver Hyndman (2014)). Dadas duas variáveis $Y_{t}$ e $X_{t}$, de modo que elas possuam uma relação de causalidade entre elas. Assim, seria possível estimar um modelo de previsão para cada variável, se utilizando dos valores defasados tanto da mesma variável quanto da outra variável e pode ser descrito através das equações (13) e (14) a seguir:

$$
\begin{aligned}
Y_{t} & =\alpha+\sum_{j=1}^{k} \beta_{j} Y_{t-j}+\sum_{j=1}^{k} \gamma_{j} X_{t-j}+u_{1 t} \\
X_{t} & =\alpha^{\prime}+\sum_{j=1}^{k} \theta_{j} X_{t-j}+\sum_{j=1}^{k} Y_{j} Y_{t-j}+u_{2 t}
\end{aligned}
$$

O índice $k$ representa as defasagens utilizadas e é definido de maneira empírica, ao se comparar modelos com valores diferentes de $k$ a partir de critérios de seleção de modelos apresentados por Akaike ou Schwarz. Deve-se levar em consideração também a quantidade total de observações, de modo a não consumir muitos de graus de liberdade.

Ainda é necessário comentar a relação deste tipo de modelo com a cointegração. Caso ela seja verificada entre as duas séries, o modelo do tipo VAR deve ser modificado para levar a característica em conta (ver Salles e Almeida (2016)), sendo adequado o modelo VAR com correção de Erros (VEC).

\subsection{Critérios de Performance e de Seleção dos Modelos}

Dentre esses critérios dois dos mais empregados são os critérios sugeridos por Akaike (AIC) e de Schwarz (BIC) cujas formulas estão descritas adiante nas expressões (15) e (16), respectivamente. Onde $\hat{\sigma}_{\varepsilon}^{2}$ representa a estimativa da variância dos termos estocásticos ou a média da soma dos quadrados dos erros. Esse procedimento é menos subjetivo do que o que se baseia na FAC e FAPC. E em algumas situações pode ser considerado um procedimento complementar ao anterior, como observado por Fava (2000).

$$
\begin{aligned}
& A I C=\ln \hat{\sigma}_{\varepsilon}^{2}+\frac{2(p+q)}{n} \\
& B I C=\ln \hat{\sigma}_{\varepsilon}^{2}+\frac{(p+q) \ln (n)}{n}
\end{aligned}
$$

Deve-se acrescentar que com amostras pequenas o AIC tende a mostrar resultados pouco confiáveis, e como alternativa pode-se utilizar o critério de Akaike corrigido (AICc) descrito, de acordo com Hyndman (2014), na equação (17) adiante, onde $p$ corresponde ao número de parâmetros do modelo analisado.

$$
A I C c=A I C+\frac{2(p+1)(p+2)}{n-p}
$$

Com os modelos obtidos foi feita uma comparação a partir de 5 critérios ou métricas de performance de modelos estocásticos. Os quatro primeiros, listados abaixo, se relacionam com a semelhança entre as observações da amostra in sample e as estimativas obtidas pelo modelo. O quinto critério utilizado, descrito pela equação (22), se refere ao somatório do quadrado da diferença entre o calculado e o existente no modelo do erro de previsão, para o período out of sample estabelecido, como descrito adiante, para se verificar o ajuste das estimativas obtidas pelos modelos ou a performance dos modelos de previsão. 


\section{SPOLM2O19}

$$
\begin{aligned}
& \text { RMSE }=\sqrt{\frac{\sum_{t=1}^{N}\left(\hat{y}_{t}-y_{t}\right)^{2}}{N}} \\
& M A E=\frac{\sum_{t=1}^{N}\left|\hat{y}_{t}-y_{t}\right|}{N} \\
& M A P E=\frac{100 \%}{N} \sum_{t=1}^{N}\left|\frac{y_{t}-\hat{y}_{t}}{y_{t}}\right| \\
& M P E=\frac{100 \%}{N} \sum_{t=1}^{N} \frac{y_{t}-\hat{y}_{t}}{y_{t}} \\
& \text { Erro }^{2}=\sum_{t=1}^{N}\left(y_{t}-\hat{y}_{t}\right)
\end{aligned}
$$

A seguir são descritos os dados utilizados.

\section{DADOS UTILIZADOS - DESCRIÇÃO DA AMOSTRA}

os As informações utilizadas neste trabalho foram de dois tipos de petróleo bruto: o Brent e o West Texas Intermediate (WTI). Enquanto o petróleo do tipo Brent é negociado na London Stock Exchange (LSE), e é a referência de preço mais utilizado mundialmente, o WTI, por sua vez, é negociado na New York Mercantile Exchange (NYMEX) e é quase exclusivo do mercado americano. Como observado por Funk (2018) o preço do WTI está associado, quase sempre, ao comportamento da economia norte-americana.

Os dados primários utilizados neste estudo são de cotações diárias do Brent e do WTI, em dólares norte-americanos por barril, e referem-se aos preços correntes, do período entre 1/01/2009 e 19/11/2018. Sendo dessa forma relativos à economia pós crise financeira deflagrada em 2008, a crise do subprime. As informações foram obtidas no web-site da U.S. Energy Information Association (EIA). A escolha do período ocorreu por ser após a eclosão da crise de 2008, que alterou as relações dos agentes econômicos e com isso as expectativas de os ativos financeiros, do petróleo e demais commodities. A partir das cotações diárias do petróleo bruto foram obtidas séries temporais semanais, que se referem as cotações do último dia de cada semana do período estudado. Com isso foi obtida uma maior homogeneidade entre os dados do WTI e Brent, uma vez que os dados diários possuem número de observações diferentes em vista da diferença de feriados ou de dias de negociações entre as bolsas NYSE e a LSE. A amostra foi dividida, com os dados a partir da data de 2/07/2017 até 19/11/2018, 15\% do número total de informações da amostra, foi formada a parte do período out of sample. Enquanto isso, a outra parte da amostra in sample foi utilizada para estimação dos modelos de previsão.

A Tabela 2, a seguir, mostra o resumo estatístico das quatros séries com os resultados dos testes de Jarque-Bera para a normalidade e os resultados dos testes ADF para estacionariedade. Pelos valores da estatística de teste do teste de Jarque-Bera, que todas as séries não obedecem ao pressuposto da normalidade. Em geral, as séries temporais de ativos financeiros não apresentam normalidade (ver Salles (2005)). Além disso, é possível observar na Tabela 2 que nenhuma das séries temporais de preços pode ser considerada estacionária. O que significa que é necessário se diferenciar essas séries ou trabalhar com a séries de variação ou retornos dos preços. As séries temporais de retornos em geral são estacionárias, 
tal como a de primeiras diferenças. O cálculo do retorno para um período t pode ser feito através da seguinte fórmula:

$$
R_{t}=\ln \left(\frac{P_{t}}{P_{t-1}}\right)
$$

Tabela 2 - Resumo Estatístico das Séries de Preços e de Retornos Utilizadas

\begin{tabular}{|c|c|c|c|c|c|c|c|c|}
\hline Estatística & $\begin{array}{l}\text { Preço } \\
\text { Brent } \\
\text { Diário }\end{array}$ & $\begin{array}{c}\text { Preço } \\
\text { WTI } \\
\text { Diário }\end{array}$ & $\begin{array}{c}\text { Preço } \\
\text { Brent } \\
\text { Semanal }\end{array}$ & $\begin{array}{c}\text { Preço } \\
\text { WTI } \\
\text { Semanal }\end{array}$ & $\begin{array}{c}\text { Retorno } \\
\text { Brent } \\
\text { Diário }\end{array}$ & $\begin{array}{c}\text { Retorno } \\
\text { WTI } \\
\text { Diário }\end{array}$ & $\begin{array}{c}\text { Retorno } \\
\text { Brent } \\
\text { Semanal }\end{array}$ & $\begin{array}{c}\text { Retorno } \\
\text { WTI } \\
\text { Semanal }\end{array}$ \\
\hline Média & 81,73 & 75,41 & 81,91 & 75,39 & 0,000057 & 0,000015 & 0,000275 & 0,000073 \\
\hline Mediana & 80,86 & 80,93 & 81,28 & 80,58 & 0,00 & 0,00 & 0,00 & 0,00 \\
\hline Máximo & 128,14 & 113,39 & 128,08 & 113,39 & 0,11 & 0,13 & 0,16 & 0,24 \\
\hline Mínimo & 26,01 & 26,19 & 28,80 & 29,39 & $-0,11$ & $-0,13$ & $-0,15$ & $-0,16$ \\
\hline Desvio Padrão & 27,81 & 22,83 & 27,81 & 22,81 & 0,02 & 0,02 & 0,04 & 0,05 \\
\hline Assimetria & $-0,15$ & $-0,31$ & $-0,15$ & $-0,29$ & 0,22 & 0,15 & $-0,27$ & 0,01 \\
\hline Curtose & 1,53 & 1,69 & 1,53 & 1,69 & 6,07 & 6,71 & 4,35 & 5,09 \\
\hline Jarque-Bera & 200,11 & 185,16 & 41,16 & 37,62 & 849,17 & 1226,59 & 38,80 & 80,28 \\
\hline Teste ADF & $-0,0349$ & $-0,4196$ & $-0,3301$ & $-0,3457$ & $-32,9765$ & $-32,9313$ & $-14,2482$ & $-15,9282$ \\
\hline$(\operatorname{Valor} P)$ & $(0,51)$ & $(0,48)$ & $(0,51)$ & $(0,51)$ & $(0,01)$ & $(0,01)$ & $(0,01)$ & $(0,01)$ \\
\hline
\end{tabular}

$\mathrm{Na}$ Tabela 2, apresentada adiante, pode-se observar que para todas as séries temporais de retornos dos preços a hipótese de estacionariedade não é rejeitada, uma vez que os valores p dos testes ADF são baixos, e assim se justifica a utilização dessas séries no decorrer deste estudo.

A seguir são apresentados os resultados obtidos com a utilização desses dados empregando-se a abordagem metodológica descrita na Seção 3.

\section{ANÁLISE DOS RESULTADOS OBTIDOS}

$\mathrm{Na}$ estimação dos modelos tipo ARIMA utilizou-se o software $\mathrm{R}$, mais especificamente com o package forecast. Após observar que as FAC e FAPC das séries não seguem as características indicadas na teoria, se seguiu para o procedimento alternativo descrito na teoria. Para cada série temporal foram analisados 44 modelos diferentes para a estimativa dos retornos dos preços, com o objetivo de se obter modelos onde todos os coeficientes, ou parâmetros, apresentaram significância estatística.

\subsection{Resultados Obtidos - Expectativas dos Retornos Diários}

A Tabela 3, adiante, mostra as estimativas dos parâmetros dos modelos ARIMA selecionados após a estimação dos modelos com as séries temporais dos retornos diários dos preços do petróleo bruto.

Para a série temporal dos retornos diários do petróleo do tipo Brent foram selecionados dois modelos onde todos os parâmetros apresentaram significância estatística, um modelo ARMA $(2,2)$ e um modelo $\operatorname{ARMA}(2,3)$. Pode-se observar na Tabela 3 que os valores do AIC e AICc são próximos em ambos os modelos. Assim como pode-se observar que os valores para o modelo $\operatorname{ARMA}(2,2)$ são menores nos critérios RMSE, MAE e MAPE. Além disso os valores dos critérios de seleção de modelos AIC e BIC são mínimos, indicando que o modelo ARMA $(2,2)$ é o mais adequado para descrever a série de retornos diários do petróleo do tipo Brent.

No que se refere a série temporal dos retornos diários do petróleo bruto do tipo WTI foram selecionados quatro modelos nos quais onde pode-se aceitar a hipótese de significância estatística de todos os parâmetros estimados, a saber, os modelos AR (1), AR (2), ARMA $(2,2)$ e ARMA $(2,3)$. Pode-se observar na Tabela 3, adiante, que dentre esses quatro modelos, o $\operatorname{AR}(1)$ e o $\operatorname{ARMA}(2,3)$ possui os melhores valores para os critérios de seleção de modelos AIC e BIC, respectivamente. 
Tabela 3 - Resultados das Estimações dos Modelos de Previsão dos Preços Diários

\begin{tabular}{|c|c|c|c|c|c|c|}
\hline Coeficientes & $\begin{array}{c}\text { Brent - Dia } \\
\text { Modelo } 1 \\
\operatorname{ARMA}(2,2)\end{array}$ & $\begin{array}{c}\text { Brent - Dia } \\
\text { Modelo } 2 \\
\operatorname{ARMA}(2,3)\end{array}$ & $\begin{array}{c}\text { WTI - Dia } \\
\text { Modelo } 3 \\
\text { AR(1) }\end{array}$ & $\begin{array}{c}\text { WTI - Dia } \\
\text { Modelo } 4 \\
\text { AR(2) }\end{array}$ & $\begin{array}{c}\text { WTI - Dia } \\
\text { Modelo } 5 \\
\text { ARMA }(2,2)\end{array}$ & $\begin{array}{c}\text { WTI - Dia } \\
\text { Modelo } 6 \\
\operatorname{ARMA}(2,3)\end{array}$ \\
\hline $\begin{array}{l}a r 1 \\
\text { (se) }\end{array}$ & $\begin{array}{c}0,2716 \\
(0,0069) \\
\end{array}$ & $\begin{array}{l}0,2721 \\
0,0058 \\
\end{array}$ & $\begin{array}{c}-0,0392 \\
0,0217\end{array}$ & $\begin{array}{c}-0,0401 \\
0,0217 \\
\end{array}$ & $\begin{array}{c}1,004 \\
0,5605 \\
\end{array}$ & $\begin{array}{c}-0,5756 \\
0,0087 \\
\end{array}$ \\
\hline $\begin{array}{l}\text { ar2 } \\
\text { (se) }\end{array}$ & $\begin{array}{c}-0,9966 \\
(0,0041) \\
\end{array}$ & $\begin{array}{c}-0,9966 \\
0,0041 \\
\end{array}$ & - & $\begin{array}{c}-0,0226 \\
0,0217 \\
\end{array}$ & $\begin{array}{c}-0,6246 \\
0,4250 \\
\end{array}$ & $\begin{array}{c}-0,9809 \\
0,0076 \\
\end{array}$ \\
\hline $\begin{array}{l}\text { ma1 } \\
(\mathrm{se})\end{array}$ & $\begin{array}{c}-0,2744 \\
(0,01070 \\
\end{array}$ & $\begin{array}{c}-0,2532 \\
0,0225 \\
\end{array}$ & - & $\begin{array}{l}- \\
- \\
\end{array}$ & $\begin{array}{c}-1,0416 \\
0,5510 \\
\end{array}$ & $\begin{array}{l}0,5326 \\
0,0225 \\
\end{array}$ \\
\hline $\begin{array}{l}\mathrm{ma2} \\
(\mathrm{se})\end{array}$ & $\begin{array}{c}0,9862 \\
(0,0093) \\
\end{array}$ & $\begin{array}{l}0,9806 \\
0,0106\end{array}$ & - & $\begin{array}{l}- \\
-\end{array}$ & $\begin{array}{l}0,6460 \\
0,4361\end{array}$ & $\begin{array}{l}0,9717 \\
0,0124 \\
\end{array}$ \\
\hline $\begin{array}{c}m a 3 \\
(\mathrm{se})\end{array}$ & $\begin{array}{l} \\
- \\
\end{array}$ & $\begin{array}{l}0,0227 \\
0,0218 \\
\end{array}$ & $\begin{array}{l}- \\
-\end{array}$ & $\begin{array}{l}- \\
- \\
\end{array}$ & $\begin{array}{l}- \\
- \\
\end{array}$ & $\begin{array}{c}-0,0426 \\
0,0216 \\
\end{array}$ \\
\hline sigma2 & 0,0004 & 0,0004 & 0,0005 & 0,0005 & 0,0005 & 0,0005 \\
\hline$R M S E$ & 0,030 & 0,030 & 0,023 & 0,023 & 0,037 & 0,034 \\
\hline$M A E$ & 0,02145 & 0,02946 & 0,01638 & 0,01641 & 0,02612 & 0,02480 \\
\hline$M P E$ & 81,006 & 80,755 & 98,30 & 98,27 & 138,00 & 75,24 \\
\hline$M A P E$ & 451,29 & 451,45 & 103,7 & 105,6 & 471,3 & 451,6 \\
\hline$A I C$ & $-10424,36$ & $-10423,43$ & $-9938,54$ & $-9937,62$ & $-9935,24$ & $-9942,36$ \\
\hline$A I C C$ & $-10424,34$ & $-10423,39$ & $-9938,53$ & $-9937,60$ & $-9935,21$ & $-9942,36$ \\
\hline$B I C$ & $-10396,06$ & $-10389,46$ & $-9927,22$ & $-9920,64$ & $-9906,95$ & $-9908,41$ \\
\hline$E{ }^{2}{ }^{2}{ }^{2}$ & 0,3495 & 0,3494 & 0,10384 & 0,10383 & 0,10435 & 0,10646 \\
\hline
\end{tabular}

Tabela 4 - Resultados das Estimações dos Modelos de Previsão dos Preços Semanais

\begin{tabular}{|c|c|c|c|}
\hline Coeficientes & $\begin{array}{c}\text { Brent - Semanal } \\
\text { Modelo } 1 \\
\text { AR(1) }\end{array}$ & $\begin{array}{c}\text { Brent - Semanal } \\
\text { Modelo } 2 \\
\operatorname{ARMA}(3,3)\end{array}$ & $\begin{array}{c}\text { WTI - Semanal } \\
\text { Modelo } 1 \\
\operatorname{ARMA}(2,2)\end{array}$ \\
\hline $\begin{array}{l}\text { ar1 } \\
\text { (se) }\end{array}$ & $\begin{array}{c}0,1074 \\
(0,0475)\end{array}$ & $\begin{array}{l}-0,1737 \\
(0,0107)\end{array}$ & $\begin{array}{l}-1,6772 \\
(0,0330)\end{array}$ \\
\hline $\begin{array}{l}\text { ar2 } \\
\text { (se) }\end{array}$ & $\begin{array}{c}-0,9809 \\
(0,0076)\end{array}$ & $\begin{array}{l}-0,1632 \\
(0,0095)\end{array}$ & $\begin{array}{l}-0,9350 \\
(0,0382)\end{array}$ \\
\hline $\begin{array}{l}\text { ar3 } \\
\text { (se) }\end{array}$ & - & $\begin{array}{l}-0,9883 \\
(0,0088)\end{array}$ & - \\
\hline $\begin{array}{l}\text { ma1 } \\
\text { (se) }\end{array}$ & - & $\begin{array}{c}0,1880 \\
(0,0127)\end{array}$ & $\begin{array}{l}0,17382 \\
(0,0201)\end{array}$ \\
\hline $\begin{array}{r}m a 2 \\
\text { (se) }\end{array}$ & - & $\begin{array}{c}0,1917 \\
(0,0166)\end{array}$ & $\begin{array}{c}0,9866 \\
(0,0269)\end{array}$ \\
\hline $\begin{array}{c}\text { ma3 } \\
\text { (se) }\end{array}$ & - & $\begin{array}{c}0,9979 \\
(0,0245)\end{array}$ & - \\
\hline sigma2 & 0,0004 & 0,0017 & 0,0022 \\
\hline$R M S E$ & 0,042 & 0,061 & 0,10 \\
\hline$M A E$ & 0,031 & 0,046 & 0,079 \\
\hline$M P E$ & 94,03 & 173,04 & 283,17 \\
\hline MAPE & 118,58 & 411,06 & 778,31 \\
\hline$A I C$ & $-1536,49$ & $-1530,41$ & $-1442,50$ \\
\hline$A I C C$ & $-1536,46$ & $-1530,15$ & $-1442,45$ \\
\hline$B I C$ & $-1528,32$ & $-1501,82$ & $-1422,16$ \\
\hline Erro $^{2}$ & 0,10 & 0,22 & 0,18 \\
\hline
\end{tabular}


Apesar de mostrar o melhor resultado do critério de Akaike, o modelo ARMA $(2,3)$ apresenta os valores mais altos para quatro das cinco métricas de performance dos modelos analisados. Por outro lado, como mostrado na Tabela 3, os modelos autoregressivos apresentam os melhores resultados dentre as cinco métricas, com um empate no critério RMSE e com o menor valor em outros dois critérios, o MAE e o MAPE para o modelo AR (1) e o MPE e erro de previsão para o modelo AR (2). Observando-se os valores dos critérios AIC e BIC, o modelo AR (1) mostra ser o melhor modelo ou o modelo mais adequado para a determinação das expectativas dos retornos diários do petróleo do tipo WTI.

\subsection{Resultados Obtidos - Expectativas dos Retornos Semanais}

A Tabela 4, acima, apresenta os resultados da estimação dos modelos ARIMA com os dados dos retornos ou variações dos preços semanais do petróleo bruto dos tipos Brent e WTI.

Para a série temporal dos retornos semanais do petróleo do tipo Brent foram selecionados dois modelos com todos os seus parâmetros estatisticamente significantes. Os dois modelos selecionados foram o AR (1) e o ARMA $(3,3)$. Pode-se observar na Tabela 4, acima, que os valores dos critérios de seleção de modelos AIC e BIC do modelo ARMA (3, 3) são maiores do que o obtido para o modelo AR (1). Além dos critérios de Akaike e Schwarz, o modelo ARMA $(3,3)$ mostra um desempenho ínfero ao modelo AR (1) nas cinco métricas de performance dos modelos. Desse modo, considera-se o modelo AR (1) o mais adequado para descrever a série de retornos semanais do petróleo tipo Brent e para se obter expectativas de retornos e, por conseguinte, dos preços do tipo Brent, que como mencionado anteriormente, corresponde a principal benchmark de preço do petróleo bruto no mercado internacional.

No que tange aos retornos semanais do petróleo tipo WTI, somente um dos 44 modelos estimados apresentou significância estatística em todos os parâmetros estimados, mostrando a dificuldade de se obter expectativas de preços dessa commodity. Desse modo, este foi o modelo escolhido, como mais adequado, para descrever e prever a série temporal em questão.

Tabela 5 - Resultados da Estimação dos Modelos VEC para retornos do Brent e WTI

\begin{tabular}{|c|c|c|c|c|c|c|c|c|}
\hline Coeficientes & $\begin{array}{c}\text { Modelo1 } \\
\text { RBrent }\end{array}$ & $\begin{array}{c}\text { dia VEC } \\
\text { RWTI }\end{array}$ & $\begin{array}{c}\text { Modelo2 } \\
\text { RBrent }\end{array}$ & $\begin{array}{c}\text { dia } V E C \\
\text { RWTI }\end{array}$ & $\begin{array}{c}\text { Modelo3 } \\
\text { RBrent }\end{array}$ & $\begin{array}{c}\text { dia VEC } \\
\text { RWTI }\end{array}$ & $\begin{array}{c}\text { Modelo1 } \\
\text { RBrent }\end{array}$ & $\begin{array}{c}\text { Sem VEC } \\
\text { RWTI }\end{array}$ \\
\hline $\begin{array}{l}\text { Cointeg. } \\
\text { (std error) }\end{array}$ & $\begin{array}{c}-0,4973 \\
(0,0291)\end{array}$ & $\begin{array}{c}0,7247 \\
(0,0313)\end{array}$ & $\begin{array}{c}-0,6174 \\
(0,0371)\end{array}$ & $\begin{array}{c}0,7879 \\
(0,0404)\end{array}$ & $\begin{array}{c}-0,6833 \\
(0,0448)\end{array}$ & $\begin{array}{c}0,9214 \\
(0,0485)\end{array}$ & $\begin{array}{c}-1,5174 \\
(0,1623)\end{array}$ & $\begin{array}{c}-0,8934 \\
(0,1826)\end{array}$ \\
\hline $\begin{array}{l}\text { RBrent } t-1 \\
\text { (std error) }\end{array}$ & $\begin{array}{c}-0,2313 \\
(0,0234)\end{array}$ & $\begin{array}{c}-0,2931 \\
(0,0251)\end{array}$ & $\begin{array}{c}-0,2257 \\
(0,0319)\end{array}$ & $\begin{array}{c}-0,4398 \\
(0,0348)\end{array}$ & $\begin{array}{c}-0,2150 \\
(0,0396)\end{array}$ & $\begin{array}{c}-0,6200 \\
(0,0429)\end{array}$ & $\begin{array}{c}0,4032 \\
(0,0145)\end{array}$ & $\begin{array}{c}0,7145 \\
(0,1634)\end{array}$ \\
\hline $\begin{array}{l}\text { RBrent }{ }_{t-2} \\
\text { (std error) }\end{array}$ & $\begin{array}{l}- \\
-\end{array}$ & $\begin{array}{l}- \\
-\end{array}$ & $\begin{array}{c}-0,1212 \\
(0,0231)\end{array}$ & $\begin{array}{c}-0,2406 \\
(0,0253)\end{array}$ & $\begin{array}{c}-0,1515 \\
(0,0324)\end{array}$ & $\begin{array}{c}-0,4556 \\
(0,0351)\end{array}$ & $\begin{array}{c}0,2968 \\
(0,1167)\end{array}$ & $\begin{array}{c}0,5091 \\
(0,1315)\end{array}$ \\
\hline $\begin{array}{l}\text { RBrent }_{t-3} \\
\text { (std error) }\end{array}$ & $\begin{array}{l} \\
-\end{array}$ & $\begin{array}{l}- \\
-\end{array}$ & $\begin{array}{l}- \\
-\end{array}$ & $\begin{array}{l}- \\
-\end{array}$ & $\begin{array}{c}-0,0884 \\
(0,0231)\end{array}$ & $\begin{array}{c}-0,2660 \\
(0,0250)\end{array}$ & $\begin{array}{c}0,1708 \\
(0,0794)\end{array}$ & $\begin{array}{c}0,2622 \\
(0,0893)\end{array}$ \\
\hline $\begin{array}{l}R W T I_{t-1} \\
\text { (std error) }\end{array}$ & $\begin{array}{c}-0,2416 \\
(0,0217)\end{array}$ & $\begin{array}{c}-0,1470 \\
(0,0233)\end{array}$ & $\begin{array}{c}-0,3772 \\
(0,0298)\end{array}$ & $\begin{array}{c}-0,1902 \\
(0,0325)\end{array}$ & $\begin{array}{c}-0,4665 \\
(0,0368)\end{array}$ & $\begin{array}{c}-0,1398 \\
(0,0398)\end{array}$ & $\begin{array}{c}-0,5565 \\
(0,1025)\end{array}$ & $\begin{array}{c}-1,0893 \\
(0,1153)\end{array}$ \\
\hline $\begin{array}{l}R W T I_{t-2} \\
\text { (std error) }\end{array}$ & $\begin{array}{l}- \\
-\end{array}$ & $\begin{array}{l}- \\
-\end{array}$ & $\begin{array}{c}-0,1963 \\
(0,0209)\end{array}$ & $\begin{array}{c}0,1102 \\
(0,0227)\end{array}$ & $\begin{array}{c}-0,3133 \\
(0,0299)\end{array}$ & $\begin{array}{c}-0,1026 \\
(0,0398)\end{array}$ & $\begin{array}{c}-0,4106 \\
(0,0901)\end{array}$ & $\begin{array}{c}-0,7878 \\
(0,1013)\end{array}$ \\
\hline $\begin{array}{l}R W T I_{t-3} \\
\text { (std error) }\end{array}$ & - & - & $\begin{array}{l}- \\
-\end{array}$ & $\begin{array}{l}- \\
-\end{array}$ & $\begin{array}{c}-0,1484 \\
(0,0299)\end{array}$ & $\begin{array}{c}-0,0576 \\
(0,0223)\end{array}$ & $\begin{array}{c}-0,2088 \\
(0,0653)\end{array}$ & $\begin{array}{c}-0,4173 \\
(0,0734)\end{array}$ \\
\hline$R^{2}$ & 0,3255 & 0,4120 & 0,3949 & 0,4575 & 0,4286 & 0,4922 & 0,4230 & 0,3603 \\
\hline SQResid & 1,2082 & 1,3925 & 1,0750 & 1,2762 & 1,0151 & 1,1891 & 0,7981 & 1,0096 \\
\hline StdError & 0,0239 & 0,0257 & 0,0226 & 0,0246 & 0,0294 & 0,0238 & 0,0432 & 0,0486 \\
\hline Stat F & 510,267 & 741,091 & 344,632 & 445,205 & 263,607 & 340,760 & 52,292 & 40,1679 \\
\hline$A I C$ & $-4,6284$ & $-4,4784$ & $-4,7428$ & $-4,5713$ & $-4,7978$ & $-4,6396$ & $-3,4308$ & $-3,1958$ \\
\hline$B I C$ & $-4,6204$ & $-4,4784$ & $-4,7295$ & $-4,5579$ & $-4,7791$ & $-4,6209$ & $-3,3652$ & $-3,1302$ \\
\hline$A I C-V E C$ & \multicolumn{2}{|c|}{$-9,3206$} & \multicolumn{2}{|c|}{$-9,4707$} & \multicolumn{2}{|c|}{$-9,5701$} & \multicolumn{2}{|c|}{$-7,8337$} \\
\hline$B I C-V E C$ & \multicolumn{2}{|c|}{$-9,2992$} & \multicolumn{2}{|c|}{$-9,4387$} & \multicolumn{2}{|c|}{$-9,5273$} & \multicolumn{2}{|c|}{$-7,6838$} \\
\hline
\end{tabular}




\subsection{Resultados dos Testes de Cointegração}

Antes de serem elaborados os dois modelos tipo VAR, é necessário testar a cointegração entre as séries, com o objetivo de identificar se o modelo deve ser um VAR ou um VAR com correção de erros, designado por VEC.

Com o teste de Engle-Granger, foram testadas as cointegrações entre as séries de retorno diárias dos preços e entre as séries temporais dos retornos semanais, assim foram feitos dois testes de cointegração. É necessário comentar que para esse teste as séries temporais devem possuir a mesma amplitude. Isso ocorre para as séries temporais semanais, enquanto as séries temporais de retornos diários possuem uma diferença de quatro observações. Assim, para esse teste e a elaboração dos modelos VAR e VEC, as últimas quatro observações da série de Brent Diário foram eliminadas. Nos resultados dos testes de cointegração, cujos resultados são exibidos na Tabela 5 acima, é visível que no primeiro caso, em que não há um deslocamento, as séries se mostram cointegradas, com o valor $\mathrm{p}$ relativo menor do que 1\%. Assim, isso indica que o modelo vetorial autoregressivo deve possuir a correção de erros, sendo assim um VEC.

\subsection{Resultados Obtidos - Modelos Vetoriais Autoregressivos - VAR e VEC}

Com a definição dos tipos dos modelos a serem utilizados, procedeu-se a estimação dos modelos vetoriais autorregressivos para as séries temporais dos retornos do preço do petróleo das tipos Brent e WTI. A determinação das defasagens dos modelos VAR e VEC foi baseada nos critérios de Akaike e Schwarz. Para ambos os casos dos retornos diários e semanais foram elaborados seis modelos, sendo os considerados para comparação os que mostraram seus todos os seus coeficientes como estatisticamente significativos.

No caso dos retornos diários, foram três, os modelos vetoriais autorregressivos com correções de erros, onde se observa todos os parâmetros como significativos. Esses modelos tem, respectivamente, defasagens iguais a 1, 2 e 3 . Ao comparar os três modelos, observa-se que os valores dos critérios de Akaike e Schwartz são muito próximos, com o modelo com três defasagens apresentando valores um pouco menores. Para a escolha do modelo mais adequado foram obtidos os valores mostrados nas Tabelas 6 e 7, apresentadas adiante, que são, respectivamente, para os retornos diários do petróleo do tipo Brent e os retornos diários do petróleo do tipo WTI. No caso dos retornos diários do petróleo do tipo Brent, o modelo VEC com $k=2$ apresenta melhores valores nos critérios RMSE e MAE. Contudo, o modelo VEC com $k=1$ demonstra possuir os melhores valores para os outros três critérios, uma vez que esses modelos possuem valores piores para os critérios AIC e BIC. O modelo com duas defasagens é o mais adequado para se obter expectativas dos retornos diários do petróleo tipo Brent. No caso do WTI, o modelo VEC com $k=2$ também apresenta os melhores valores para dois critérios, contudo o VEC de uma defasagem apresenta valores melhores nos outros três critérios. Ao se considerar que o VEC com duas defasagens apresenta melhores valores de AIC e BIC, deve-se apontar como mais adequado para representar a série temporal dos retornos diários dos preços do petróleo do tipo WTI.

Tabela 6 - Comparação dos modelos VEC para retornos do Brent diário

\begin{tabular}{l|c|c|c|c|c|c|c}
\hline & RMSE & MAE & MPE & MAPE & Erro $^{2}$ & AIC & BIC \\
\hline VEC $k=1$ & 0,050 & 0,046 & 61,87 & 101,82 & 0,16 & -9320 & -9299 \\
VEC $k=2$ & 0,032 & 0,027 & 64,55 & 111,52 & 0,18 & -9470 & -9438 \\
VEC $k=3$ & 0,038 & 0,034 & 77,68 & 104,64 & 0,20 & -9570 & -9527 \\
\hline
\end{tabular}

Tabela 7 - Comparação dos modelos VEC para retornos do WTI diário

\begin{tabular}{l|c|c|c|c|c|c|c}
\hline & RMSE & MAE & MPE & MAPE & Erro $^{2}$ & AIC & BIC \\
\hline VEC $k=1$ & 0,049 & 0,045 & $-37,20$ & 103,03 & 0,18 & -9320 & -9299 \\
VEC $k=2$ & 0,034 & 0,028 & 54,65 & 113,75 & 0,20 & -9470 & -9438 \\
VEC $k=3$ & 0,041 & 0,036 & 59,15 & 106,06 & 0,25 & -9570 & -9527 \\
\hline
\end{tabular}


Para o caso dos retornos semanais dos preços, um dos modelos autorregressivos analisados se mostrou adequado. Esse modelo é o com defasagem igual a 3, e pode ser observado na Tabela 5, mostrada anteriormente. Por ser o único a apresentar os coeficientes estatisticamente significativos, é o modelo escolhido para as previsões dos retornos semanais do petróleo do tipo Brent e do petróleo do tipo WTI.

\subsection{Comparação dos Resultados Obtidos: Modelos ARIMA e VEC}

Cabe observar se existe ganho de informação com os modelos VEC em relação aos modelos ARIMA obtidos anteriormente. Isso foi feito para cada uma das quatro séries temporais dos retornos diários do petróleo bruto , como mostrado a seguir. Para o caso dos retornos diários do petróleo bruto do tipo Brent é possível observar que o modelo ARMA (2, 2) se mostra inferior em três das cinco métricas utilizadas para comparar a performance dos modelos selecionados. Contudo, ele possui os valores mais negativos para os critérios de Akaike e Schwarz, e mostra valores melhores de RMSE e MAE, aparentando que não tenha ocorrido uma melhora significativa com o modelo VEC. A comparação se encontra na Tabela 8, abaixo. Enquanto no caso dos retornos diários do WTI, o modelo AR (1) aparenta ser mais adequado do que o modelo vetorial em quatro dos critérios, apesar de possuir quase o dobro do MPE, como mostra a Tabela 9, adiante. Além disso, ele possui valores mais negativos do AIC e BIC. Desse modo, é possível afirmar que não ocorreu uma melhora significante com o modelo VEC.

E no caso dos retornos semanais, os modelos relativos aos preços do petróleo bruto do tipo Brent se encontram na Tabela 10. Pode-se observar nesta Tabela 10 que o modelo AR (1) é o mais adequado em todas as cinco métricas de performance, quando se compara com o modelo VEC. Desse modo, é possível se observar que não houve uma melhora significativa com os modelos vetoriais autoregressivos, sendo o modelo AR (1) o que obteve melhores valores das métricas de performance de modelos.

Tabela 8 - Comparação dos modelos ARIMA e VEC para retornos do Brent diário

\begin{tabular}{l|c|c|c|c|c|c|c}
\hline & RMSE & MAE & MPE & MAPE & Erro $^{2}$ & AIC & BIC \\
\hline ARMA(2,2) & 0,029 & 0,021 & 81,01 & 451,29 & 0,35 & -10424 & -10396 \\
VEC $k=2$ & 0,032 & 0,027 & 64,55 & 111,52 & 0,18 & -9470 & -9438 \\
\hline
\end{tabular}

Tabela 9 - Comparação dos modelos ARIMA e VEC para retornos do WTI diário

\begin{tabular}{l|c|c|c|c|c|c|c}
\hline & RMSE & MAE & MPE & MAPE & Erro $^{2}$ & AIC & BIC \\
\hline AR(1) & 0,023 & 0,016 & 98,30 & 103,70 & 0,10 & -9938 & -9927 \\
VEC $k=2$ & 0,034 & 0,028 & 54,65 & 113,75 & 0,20 & -9470 & -9438 \\
\hline
\end{tabular}

Tabela 10 - Comparação dos modelos ARIMA e VEC para retornos do Brent Semanal

\begin{tabular}{l|c|c|c|c|c|c|c}
\hline & RMSE & MAE & MPE & MAPE & Erro $^{2}$ & AIC & BIC \\
\hline ARMA(2,2) & 0,042 & 0,031 & 94,03 & 118,58 & 0,10 & -1536 & -1528 \\
VEC $k=2$ & 0,043 & 0,032 & 161,17 & 457,37 & 0,12 & -7833 & -7683 \\
\hline
\end{tabular}

Tabela 11 - Comparação dos modelos ARIMA e VEC para retornos do WTI semanal

\begin{tabular}{l|c|c|c|c|c|c|c}
\hline & RMSE & MAE & MPE & MAPE & Erro $^{2}$ & AIC & BIC \\
\hline ARMA(2,2) & 0,100 & 0,079 & 283,17 & 778,31 & 0,18 & -1442 & -1422 \\
VEC $k=3$ & 0,046 & 0,036 & 153,24 & 300,81 & 0,12 & -7833 & -7683 \\
\hline
\end{tabular}

Por fim, os modelos para os retornos do WTI semanal são comparados na Tabela 11, acima. É visível que, neste caso, ocorre uma melhora com os modelos VEC, em comparação com o modelo $\operatorname{ARMA}(2,2)$. Esse resultado pode ser derivado da dificuldade em obter o modelo ARIMA com todos os parâmetros significativos para os retornos semanais do WTI, uma vez que, dentre os 44 analisados, somente um se mostrou como opção e, assim, foi o utilizado. A superioridade dos resultados do modelo VEC ocorre 
também para o caso dos critérios AIC e BIC. Desse modo, o modelo vetorial autoregressivo se mostra melhor, e há um ganho de informação.

\section{COMENTÁRIOS FINAIS}

Este trabalho teve como propósito estudar a adequação de modelos clássicos de previsão do tipo ARIMA para obtenção de expectativas dos preços do petróleo bruto dos tipos Brent e WTI para o curto ou curtíssimo prazo. Esses modelos se mostraram viáveis para a formação das expectativas dos preços de petróleo dos dois principais benchmarks do preço do petróleo bruto negociado no mercado internacional selecionados para este trabalho, o Brent e o WTI. Deve-se observar que dessa forma este estudo contribui para a necessária discussão sobre formação das expectativas do preço de petróleo bruto, variável importante para a tomada de decisões dos diversos agentes econômicos que participam direta ou indiretamente.

A revisão da literatura, em um resumo aqui apresentado, mostrou uma diversidade de métodos econométricos utilizados, destacando a relevância e a complexidade do tema, ou seja, da busca por modelos de maior adequação e acurácia para previsão dos preços de commodities, em particular, dos preços do petróleo bruto, no curto ou curtíssimo prazo. Deve-se perceber, assim, a existência de uma complexidade ainda maior no que se refere determinação de expectativas para longo prazo, muitas vezes necessárias e muitas vezes incertas. Enquanto as pesquisas procuram estabelecer expectativas futuras de preços, o futuro se mostra incerto. Uma vez que são muitas as variáveis estocásticas que influem no comportamento desses preços, aumentando a dificuldade dos estudos relacionados.

Deve-se destacar que este estudo se diferencia dos demais apresentados na literatura econométrica e de economia de energia, por se utilizar de uma amostra com dados posteriores a deflagração da crise financeira do subprime em 2008. No entanto os resultados aqui apresentados devem ser vistos com ressalvas pois, embora a hipótese de estacionariedade não tenha sido rejeitada, os dados utilizados violam o pressuposto da normalidade.

Como sugestão para futuros trabalhos sobre o tema deve-se sugerir uma alternativa para o problema da não normalidade dos dados relativos aos preços do petróleo bruto. Assim como deve-se procurar utilizar modelos da família ARCH, ou de volatilidade estocástica, para tratar a volatilidade dos preços do petróleo bruto ou dos benchmarks aqui estudados. A utilização de modelos que levam em consideração a heteroscedasticidade dos preços de commodities é algo frequente na literatura, e que deve ser explorada no contexto do pós crise financeira do subprime. Outros tópicos que podem ser considerados, em trabalhos futuros sobre o tema aqui tratado, são a sazonalidade dos preços e a interação com as diversas variáveis macroeconômicas que o influenciam os preços do petróleo bruto.

\section{REFERÊNCIAS BIBLIOGRÁFICAS}

[1] Braginskii, O. (2009), Crude oil prices: History, forecast, and impact on economy, Russian Journal of General Chemistry, v. 79, n. 11, 2486-2498.

[2] Behmiri, N., Manso, J. (2013), Crude Oil Price Forecasting Techniques: A Comprehensive Review of Literature, SSRN Electronic Journal, 30-48.

[3] Worthington, A. (2012), A State-of-the-Art Review of Finance Research in Physical and Financial Trading Markets in Crude Oil Exploration in the World, 203-220.

[4] Frey, G. (2009) Econometric Models for Oil Price Forecasting: A Critical Survey, CESifo Forum, v. 1, p. 29-44. 
[5] Assis, K., Amran, A., Remali, Y. (2010), Forecasting Cocoa Bean Prices Using Univariate Time Series Models, Journal of Arts, Science \& Commerce, v. 1, n. 1, 71-80.

[6] Nadarajah, S., Afuecheta, E., Chan, S. (2014), GARCH modeling of five popular commodities, Empirical Economics, v. 48, n. 4, 1691-1712.

[7] Klein, T., Walther, T. (2016), Oil price volatility forecast with mixture memory GARCH, Energy Economics, v. 58, 46-58.

[8] Wang, Y., Liu, L., Wu, C. (2017), Forecasting the real prices of crude oil using forecast combinations over time-varying parameter models, Energy Economics, v. 66, 337-348.

[9] Funk, C. (2018), Forecasting the real price of oil - Time-variation and forecast combination, Energy Economics, v. 76, 288-302.

[10] Herrera, A., Hu, L., Pastor, D. (2018), Forecasting crude oil price volatility, International Journal of Forecasting, v. 34, n. 4, 622-635.

[11] Dbouk, W., Jamali, I. (2018), Predicting daily oil prices: Linear and non-linear models. Research in International Business and Finance, v. 46, 149-165.

[12] Gujarati, D., Porter, D. (2011) Econometria Básica, 5ª ed., São Paulo, AMGH Editora Ltda.

[13] Fava, V. L. (2000), Metodologia de Box-Jenkins para Modelos Univariados, in Vasconcellos, M. A., Manual de Econometria, Editora Atlas.

[14] Hyndman, R. (2014), Forecasting: Principles \& Practice. University of Western, Australia.

[15] Salles, A. A., Almeida, P. H. A. (2017), The Crude Oil Price Influence on the Brazilian Industrial Production, Open Journal of Business and Management, 5, 401414.

[16] Salles, A. A. (2005), Notas de Aula de Métodos Quantitativos Aplicados a Finanças Notas de Aula, Escola Politécnica, Universidade Federal do Rio de Janeiro. 